\title{
A Significance Measure for R-Fuzzy Sets
}

\author{
Arjab Singh Khuman \& Yingjie Yang \\ Centre for Computational Intelligence \\ School of Computing \\ De Montfort University, \\ Leicester, United Kingdom
}

\author{
Robert John \\ Automated Scheduling, Optimisation \& Planning Group \\ School of Computer Science \\ University of Nottingham, \\ Nottingham, United Kingdom
}

\begin{abstract}
This paper presents a newly created significance measure based on a variation of Bayes' theorem, one which quantifies the significance of any value contained within an R-fuzzy set. An R-fuzzy set is a relatively new concept and an extension to fuzzy sets. By utilising the lower and upper approximations from rough set theory, an R-fuzzy approach allows for uncertain fuzzy membership values to be encapsulated. The membership values associated with the lower approximation are regarded as absolute truths, whereas the values associated with the upper approximation maybe be the result of a single voter, or the vast majority, but definitely not all. By making use of the significance measure one can inspect each and every encapsulated membership value. The significance value itself is a coefficient, this value will indicate how strongly it was agreed upon by the populace for a specific R-fuzzy descriptor. There has been no recent effort made in order to make sense of the significance of any of the values contained within an R-fuzzy set, hence the motivation for this paper. Also presented is a worked example, demonstrating the coupling together of an Rfuzzy approach and the significance measure.
\end{abstract}

\section{INTRODUCTION}

The work presented by Yang and Hinde in [12], was the first work that proposed the concept of an R-fuzzy set. Yet another approach to encapsulate uncertainty, but the first one which adopted rough approximations for the bounding of uncertain fuzzy membership values. The membership value of an R-fuzzy set, is itself a set, more specifically a rough set. This encapsulation is bounded by crisp approximations; the use of lower and upper approximations directly from rough set theory. The lower bound contains the membership values agreed upon by all, whereas the upper bound contains membership values agreed upon by at least one voter. There are several existing paradigms and concepts related to uncertainty, all of which have their own associated difficulties in extracting, crisp, clear and concise information. The foundational understanding of sets from a classical sense is with regards to absolute inclusion, or absolute exclusion from the set [2]. With relation to human inferencing, using such a precise and crisp manner did not seem a fitting synthesis, everything is vague to some extent. With this realisation came the notion of mereology, which played on the ideals of an element having partial belongingness to a set. For precise reasoning a crisp understanding is needed, but this becomes problematic when considering natural language. As is evident in our daily communications, we are often obliged to use words which are themselves associated with inherent vagueness and ambiguity. Therefore, to mimic and understand human based reasoning, an entirely crisp, classical use of logic can not solely be used. The paradigm of fuzzy theory, which apart of its repertoire includes fuzzy logic [13][14], adopts the notion of mereology. Whereby an element can be attributed to belongingness to some degree; a degree of membership inclusive of its membership function.

One problem that is still prevalent, is deriving a crisp membership function for a type-1 fuzzy set, as it may still involve a great deal a vagueness and ambiguity [12]. This has led to various extensions and hybridisations, all in the hope to alleviate this concern. Such concepts include; Atanassov intuitionistic fuzzy sets [1], where a degree of membership and degree of non-membership are presented. Shadowed sets [10], where the evaluation of a membership is scored as either $(1),(0)$ or belonging to the shadowed region $[0,1]$. Interval-valued fuzzy sets [11], where the membership of an individual element is characterised as an interval itself. Type-2 fuzzy sets [5], where the secondary grade membership function itself is a type-1 fuzzy set. These new approaches involve the use of intervals, multiple parameters and additional fuzzy sets to describe the uncertain membership function values of fuzzy sets. However, there is still a problem, these approaches will not be able to distinguish between the values contained within their intervals or shadow regions. As R-fuzzy utilises rough set approximations, all values contained in the populace are included, thus conserving general and specific consensus. There is no loss of information and every value is easily distinguishable. Rough sets provide for a different perspective to that of fuzzy sets, with relation to how uncertainty is handled. A rough approach is with regards to ambiguity and a lack of information. Whereas, a fuzzy approach is more associated to vagueness and a lack of definable boundaries. As a result there have been several hybridisations between fuzzy sets and rough sets; [3][4][6], all of which mainly involve the use of equivalence and similarity relations. R-fuzzy is yet another approach which pairs fuzzy and rough aspects together, but it was the first to use rough sets to approximate the membership functions of fuzzy sets [12].

Section II will describe the preliminaries for approximations and R-fuzzy sets. Section III presents the newly created significance measure. Also included is a worked example involving human-based visual perception, coupling together the R-fuzzy approach with the significance measure. Section IV concludes and summarises the advantages of using an Rfuzzy approach, paired with a significance measure for humanbased perception modelling.

\section{PRELIMINARIES}

This paper will now present the definitions and notations used, we begin with approximations. It is assumed that the 
reader will be well-versed in the foundational aspects of fuzzy and rough sets, hence why their definitions have been omitted from this paper.

\section{A. Approximation Preliminaries}

Definition 1 (Approximations [8][9]): Assume that $\Lambda=$ $(\mathbb{U}, A)$ is an information system and that $B \subseteq A$ and $X \subseteq \mathbb{U}$. One can approximate set $X$ with the information contained in $B$ via a lower and upper approximation set.

The lower approximation is the set of all objects that absolutely belong to set $X$ with respect to $B$. It is the union of all equivalence classes in $[x]_{B}$ which are contained within the target set $X$, and is given by the formal expression:

$$
\begin{gathered}
\underline{B} X=\left\{x \mid[x]_{B} \subseteq X\right\} \\
\underline{B}(x)=\bigcup_{x \in \mathbb{U}}\{B(x): B(x) \subseteq X\}
\end{gathered}
$$

The upper approximation is the set of all objects which can be classified as being possible members of set $X$ with respect to $B$. It is the union of all equivalence classes that have a non-empty intersection with the target set $X$, and is given by the formal expression:

$$
\begin{gathered}
\bar{B} X=\left\{x \mid[x]_{B} \cap X \neq \emptyset\right\} \\
\bar{B}(x)=\bigcup_{x \in \mathbb{U}}\{B(x): B(x) \cap X \neq \emptyset\}
\end{gathered}
$$

\section{B. R-Fuzzy Set Preliminaries}

We now present the concept of R-fuzzy sets, which makes use of the approximations as given in Definition 1.

Definition 2 (R-fuzzy sets [12]): Let the pair apr = $\left(J_{x}, B\right)$ be an approximation space on a set of values $J_{x}=$ $\left\{v_{1}, v_{2}, \ldots, v_{n}\right\} \subseteq[0,1]$, and let $J_{x} / B$ denote the set of all equivalence classes of $B$. Let $\left(\underline{M}_{A}(x), \bar{M}_{A}(x)\right)$ be a rough set in $a p r$. An R-fuzzy set $A$ is characterised by a rough set as its membership function $\left(\underline{M}_{A}(x), \bar{M}_{A}(x)\right)$, where $x \in \mathbb{U}$, given by the formal expression:

$$
\begin{gathered}
A=\left\{\left\langle x,\left(\underline{M}_{A}(x), \bar{M}_{A}(x)\right)\right\rangle \mid\right. \\
\left.\forall x \in \mathbb{U}, \underline{M}_{A}(x) \subseteq \bar{M}_{A}(x) \subseteq J_{x}\right\} \\
A=\sum_{x \in \mathbb{U}}\left(\underline{M_{A}}(x), \bar{M}_{A}(x)\right) / x
\end{gathered}
$$

Where $\sum$ denotes the union of all admissible $x$ elements over the universe of discourse. For each $x_{i} \in \mathbb{U}$, there is an associated membership description $d\left(x_{i}\right)$ which describes the belongingness of the element $x_{i}$ to the set $A \subseteq \mathbb{U}$. Assume $C$ is a set of available evaluation criteria. Each value $v \in J_{x}$ is evaluated by $c_{j} \in C$ to determine if it is described by the membership description for $x_{i}$ with respect to $A$. The resulting evaluation will either be a YES or a NO. Typically, evaluations which result in a NO are ignored and only evaluations that result in a YES are carried forward. If a membership value has an association to the descriptor that the R-fuzzy set is being modelled for, it will be included within that R-fuzzy set.

$$
v \stackrel{\left(d\left(x_{i}\right), c_{j}\right)}{\longrightarrow} \text { YES } \quad \text { or } \quad v \stackrel{\left(d\left(x_{i}\right), c_{j}\right)}{\longrightarrow} \mathrm{NO}
$$

For each pair $\left(\left(x_{i}\right), c_{j}\right)$ where $x_{i} \in \mathbb{U}$ and $c_{j} \in C$, a set $M_{c j}\left(x_{i}\right) \subseteq J_{x}$ is created, given by the formal expression:

$$
M_{c j}\left(x_{i}\right)=\left\{v \mid v \in J_{x}, v \stackrel{\left(d\left(x_{i}\right), c_{j}\right)}{\longrightarrow} \mathrm{YES}\right\}
$$

The lower approximation of the rough set $M\left(x_{i}\right)$ for the membership function described by $d\left(x_{i}\right)$ is given by:

$$
\underline{M}\left(x_{i}\right)=\bigcap_{j} M_{c j}\left(x_{i}\right)
$$

The upper approximation of the rough set $M\left(x_{i}\right)$ for the membership function described by $d\left(x_{i}\right)$ is given by:

$$
\bar{M}\left(x_{i}\right)=\bigcup_{j} M_{c j}\left(x_{i}\right)
$$

The rough set approximating the membership $d\left(x_{i}\right)$ for $x_{i}$ is given as:

$$
M\left(x_{i}\right)=\left(\bigcap_{j} M_{c j}\left(x_{i}\right), \bigcup_{j} M_{c j}\left(x_{i}\right)\right)
$$

Considering, $v \stackrel{\left(d\left(x_{i}\right), c_{j}\right)}{\longrightarrow}$ YES, it is perfectly possible for a different criteria set $C$ to produce an entirely different $M_{c j}\left(x_{i}\right)$ and hence an entirely different $M\left(x_{i}\right)$. This means that an Rfuzzy set can only be created when a membership set $J_{x}$ and criteria set $C$ have already been established [12].

\section{SignifiCANCE MEAsure}

This section will present the newly derived significance measure. Also included is a worked example, demonstrating the pairing of an R-fuzzy and significance measure approach.

\section{A. Significance}

The significance measure proposed in this paper is based on the certainty factor employed by rough set theory, which itself is based on a variation of Bayes' theorem [8]. The certainty factor presented from a rough set perspective is given as follows:

$$
\operatorname{cer}_{x}(A, D)=\frac{|A(x) \cap D(x)|}{|A(x)|}
$$

For every decision rule $A \rightarrow_{x} D$, there is an associated certainty factor, where $A$ is the rule, $D$ is the decision and supp is a frequency count. Eq. (8) can be viewed as a conditional probability that $y$ belongs to $D(x)$ given that $y$ belongs to $A(x)$. If $\operatorname{cer}_{x}(A, D)=1$, then $A \rightarrow_{x} D$ will be called a certain decision rule, if $0<\operatorname{cer}_{x}(A, D)<1$ the decision rule will be referred to as an uncertain decision rule. As Rfuzzy sets are not used on rule based systems, one cannot simply transfer over the certainty factor without modification. 
The significance measure must be made relative to the subset of all values based on $M_{p j}(x) \subseteq J_{x}$.

Definition 3 (Degree of significance): Using the same notations given in Definition 2 that described an R-fuzzy set, assume that an R-fuzzy set $M\left(x_{i}\right)$ has already been created, and that a membership set $J_{x}$ and a criteria set $C$ are also known. Given that $|N|$ is the cardinality of all generated subsets $M_{c j}\left(x_{i}\right)$, and that $S_{v}$ is the number of subsets that contain the specified membership value being inspected. As each value $v \in J_{x}$ is evaluated by $c_{j} \in C$, the significance measure therefore counts the number of instances that $v$ occurred over $|N|$, given by the formal expression:

$$
\gamma_{\bar{A}}\{v\}=\frac{S_{v}}{|N|}
$$

The significance measure expresses the conditional probability that $v \in J_{x}$ belongs to the R-fuzzy set $M\left(x_{i}\right)$, given by its descriptor $d\left(x_{i}\right)$. The value will initially be presented as a fraction, where the denominator $|N|$ will be indicative of the total number of subsets. The numerator $S_{v}$ will be the number of occurrences, that the observed membership value was accounted for. This fraction in turn can be translated into a normalised real number, which will be indicative of its significance and given by its membership function $\gamma_{\bar{A}}\{v\}$ : $J_{x} \rightarrow[0,1]$. If the value returned by $\gamma_{\bar{A}}\{v\}=1$, then that particular membership value has been agreed upon by all in the criteria set $C$. As a result one will know that it absolutely belongs to the lower approximation; as for it to be included, the entire populace must agree:

$$
\underline{M}_{A}=\left\{\gamma_{\bar{A}}\{v\}=1 \mid v \in J_{x} \subseteq[0,1]\right\}
$$

The notation for an R-fuzzy set given by Eq. (3), indicated that a lower approximation was a subset of the upper approximation. Therefore, any membership value with a significance degree of $\gamma_{\bar{A}}\{v\}=1$, will also be included within the upper approximation. If $\gamma_{\bar{A}}\{v\}=0$, this particular membership value will be disregarded as it does not satisfy the descriptor at all. If $0<\gamma_{\bar{A}}\{v\}<1$, then this particular membership value has some significance to some degree relative to the descriptor $d\left(x_{i}\right)$. As a result this particular value will knowingly be contained within the upper approximation as its significance is greater than 0 :

$$
\bar{M}_{A}=\left\{\gamma_{\bar{A}}\{v\}>0 \mid v \in J_{x} \subseteq[0,1]\right\}
$$

The interpretation of the membership value from a significance measure perspective, is different to that from a fuzzy perspective. A fuzzy element can be described by its membership function such that it returns any real number in the range $[0,1]$, implying its belongingness to its fuzzy set. The significance measure however returns the degree of significance of a membership value relative to its descriptor $d\left(x_{i}\right)$. This is based on its conditional probability of distribution.

Eq. (9) can be rewritten so that the collected significance degrees constitute a set, given by the following expression:

$$
\begin{gathered}
\bar{A}=\left\{\left\langle v, \gamma_{\bar{A}}\{v\}\right\rangle \mid v \in J_{x} \subseteq[0,1]\right\} \\
\bar{A}=\sum_{v \in J_{x}} \gamma_{\bar{A}}\{v\} / v
\end{gathered}
$$

Where $\bar{A}$ is a set describing the distribution of a specified descriptor $d(x)$, for which the generated R-fuzzy set was created for. It's noteworthy to mention that fuzzy sets are not associated with probability, merely the degree of inclusion. As the newly derived significance measure that this paper proposes is based on Eq. (8), the significance is indeed associated with the conditional probability of distribution. The greater the returned value for $\gamma_{\bar{A}}\{v\}$ the greater its significance with regards to the descriptor that the R-fuzzy set is being modelled for. By inferring from the returned degree of significance for any membership value, one can use this information to better understand the populace it was generated from. R-fuzzy sets allow for every conceivable perception to be incorporated, that includes all possible outliers. The associated degrees of significance will quantify just how important, or unimportant a membership value truly is based on the perceptions collected.

Example 1: Assume that $F=\left\{f_{1}, f_{2}, \ldots, f_{9}\right\}$ is a set containing 9 different colour swatches, all of which are a variation of the colour red:

$$
\begin{aligned}
& f_{1} \rightarrow \quad[204,0,0] \rightarrow \\
& f_{2} \rightarrow \quad[153,0,0] \rightarrow \\
& f_{3} \rightarrow \quad[255,102,102] \rightarrow \\
& f_{4} \rightarrow \quad[51,0,0] \rightarrow \\
& f_{5} \rightarrow \quad[255,153,153] \rightarrow \\
& f_{6} \rightarrow \quad[102,0,0] \rightarrow \\
& f_{7} \rightarrow \quad[255,204,204] \rightarrow \\
& f_{8} \rightarrow \quad[255,0,0] \rightarrow \\
& f_{9} \rightarrow \quad[255,51,51] \rightarrow
\end{aligned}
$$

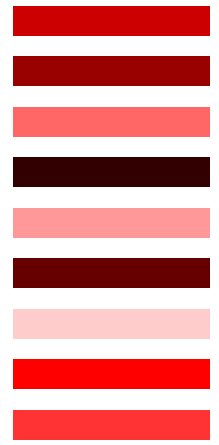

The colours themselves are given by their [RGB] values, from which the average is taken for each one and passed to $N$. The values contained are given as $N=$ $\{68,51,153,17,187,34,221,85,119\}$. Each average $N_{i}$ value will correspond to a specific colour swatch $F_{i}$. For example, the swatch associated with $f_{1}$ has a value of $68, f_{2}$ will be related to 51, and so on. Assume that the criteria set $C=\left\{p_{1}, p_{2}, \ldots, p_{6}\right\}$ contains the perceptions of 6 individuals, all of whom gave their own opinions based on the available descriptors and the swatches themselves. These values have been collected and are presented in Table I.

TABLE I. HUMAN PERCEPTION BASED ON THE VARIATIONS FOR THE COLOUR RED

\begin{tabular}{c|ccccccccc}
\cline { 2 - 10 } & $f_{1}$ & $f_{2}$ & $f_{3}$ & $f_{4}$ & $f_{5}$ & $f_{6}$ & $f_{7}$ & $f_{8}$ & $f_{9}$ \\
\hline \hline$p_{1}$ & $D R$ & $D R$ & $L R$ & $D R$ & $L R$ & $D R$ & $L R$ & $R$ & $R$ \\
$p_{2}$ & $R$ & $D R$ & $R$ & $D R$ & $L R$ & $D R$ & $L R$ & $R$ & $R$ \\
$p_{3}$ & $R$ & $D R$ & $L R$ & $D R$ & $L R$ & $D R$ & $L R$ & $R$ & $L R$ \\
$p_{4}$ & $D R$ & $R$ & $L R$ & $D R$ & $L R$ & $D R$ & $L R$ & $R$ & $L R$ \\
$p_{5}$ & $D R$ & $D R$ & $R$ & $D R$ & $L R$ & $D R$ & $L R$ & $R$ & $L R$ \\
$p_{6}$ & $D R$ & $D R$ & $L R$ & $D R$ & $L R$ & $D R$ & $L R$ & $R$ & $R$ \\
\hline
\end{tabular}

The terms contained within the table can be understood as meaning:

$$
L R \rightarrow \text { Light Red } \quad R \rightarrow \text { Red } \quad D R \rightarrow \text { Dark Red }
$$

One must now create a fuzzy membership set $J_{x}$. As it was stated in Definition 2, the criteria set $C$ and membership 
set $J_{x}$ must be made available as a prerequisite, so that an R-fuzzy set can be established. Applying the values contained in $N$ on the linear function given in Eq. (13):

$$
\mu\left(f_{i}\right)=\frac{N_{i}-N_{\min }}{N_{\max }-N_{\min }}
$$

One is then able to obtain a fuzzy membership set, the resulting fuzzy membership set is given as follows:

$$
J_{x}=\{0.25,0.17,0.67,0.00,0.83,0.08,1.00,0.33,0.50\}
$$

It is not always possible to know the exact RGB value of a particular colour swatch, nor do people have to know the exact value in their communications with one another [12]. An R-fuzzy approach provides an answer to the question, how to express a fuzzy membership function if the exact colour saturation levels are not known? Assume that a $10^{\text {th }}$ colour swatch is presented to the populace, and has an associated descriptor of being Red $(R)$, what fuzzy membership value or values would one assign to this swatch? A traditional fuzzy approach would give an exact value, however, as Table I shows, individuals can give different perceptions for the same initial observation. What maybe perceived as Red to some may not be Red to all. R-fuzzy allows for any membership value with an affinity to the descriptor it is being modelled for, to be included within its membership set. Thus allowing for a general consensus and also individual nuances.

Assuming that the $10^{\text {th }}$ colour swatch $f_{10}$ has been been described as being Red, the descriptor can be set to $d\left(f_{10}\right)=$ 'Red'. Based on what has already been collected in Table I, any value that correlates with $R$, will provide for a valid membership value. Each individual from the criteria set $C$ is inspected, such that each value $v \in J_{x}$ is evaluated against $p_{j} \in C$ to conclude if it fits with the description given for $d\left(f_{10}\right)$, and for $f_{10} \in \mathbb{U}$, using:

$$
v \stackrel{\left(d\left(x_{i}\right), c_{j}\right)}{\longrightarrow} \text { YES }
$$

For each $p_{i} \in C$ there is a corresponding row in Table I. For the columns where there is a match with the descriptor given for $d\left(f_{10}\right)$, its corresponding colour swatch $f_{i}$ will be used to provide the membership values. Using Red as the descriptor for swatch $f_{10}$ and the perceptions given in Table I, one can create a subset of values $M_{p j}\left(f_{11}\right) \subseteq J_{x}$. For example, take $p_{1}$, one can infer that swatches $f_{8}$ and $f_{9}$ were the only swatches that satisfy the descriptor when set to $d\left(f_{10}\right)=$ 'Red'. Therefore, the correlating membership values for $f_{8}$ and $f_{9}$ provide the subset $M_{p 1}\left(f_{10}\right)=\{0.33,0.50\}$. This is repeated for all observations contained in the criteria set $C$, the results of which are given as follows:

$$
\begin{aligned}
& M_{p 1}\left(f_{10}\right)=\{0.33,0.50\} \\
& M_{p 2}\left(f_{10}\right)=\{0.25,0.33,0.50,0.67\} \\
& M_{p 3}\left(f_{10}\right)=\{0.25,0.33\} \\
& M_{p 4}\left(f_{10}\right)=\{0.17,0.33\} \\
& M_{p 5}\left(f_{10}\right)=\{0.33,0.67\} \\
& M_{p 6}\left(f_{10}\right)=\{0.33,0.50\}
\end{aligned}
$$

Based on the individual subsets collected $M_{p j}\left(f_{10}\right)$, one can now apply the concept of approximations as given in
Definition 1. Beginning with the lower approximation given by Eq. (5), each subset is inspected to find if any membership value from $J_{x}$ occurred in each and every subset. One can conclude that the fuzzy membership value $\{0.33\}$ was the only value that was agreed upon by all, and that it satisfied the descriptor $d\left(f_{10}\right)=$ 'Red'. As a result $\{0.33\}$ is the only value contained in the lower approximation. If no such value was agreed upon by all, then the lower approximation would remain empty. The upper approximation given by Eq. (6), can be understood as containing values that have been considered by at least one voter, such that it satisfies the descriptor. Therefore, all instances contained within the generated subsets will also be contained in the upper approximation: $\{0.17,0.25,0.33,0.50,0.67\}$, notice the removal of duplicated elements.

The actual rough set approximating the uncertain membership for $d\left(f_{10}\right)$ is constructed using Eq. (7), the result of which is given as follows:

$$
M\left(f_{11}\right)=(\{0.33\},\{0.17,0.25,0.33,0.50,0.67\})
$$

Therefore, the membership value 0.33 was the only fuzzy membership that was agreed upon, as being a suitable value for when describing the descriptor Red. Referring to Table I, one can see that the swatch for $f_{8}$ was the only colour that was agreed upon unanimously. It correlates to the swatch with an RGB value of $[255,0,0]$, which from a digital perspective is absolutely red. Also, the swatches associated with the memberships $0.17,0.25,0.50$ and 0.67 were also considered Red by some, but not all. It is a this point that the significance measure would be beneficial, as it will be able to obtain the importance of each and every value contained in the R-fuzzy set. Using Eq. (9) one can apply a significance coefficient to each of the membership values contained in the membership set $J_{x}$. The greater the value the greater its significance in relation to its descriptor, and the more individuals that agreed with its sentiment. This is achieved by simply counting the number of occurrences that each inspected membership occurred throughout all the generated subsets $M_{p j}\left(f_{10}\right)$. As a result, we can obtain the following significance measure values for each of the membership values associated when the descriptor is set to Red.

$$
\begin{array}{ll}
\gamma_{\bar{R}}\{0.00\}=\frac{0}{6}=0.00 & \gamma_{\bar{R}}\{0.08\}=\frac{0}{6}=0.00 \\
\gamma_{\bar{R}}\{0.17\}=\frac{1}{6}=0.17 & \gamma_{\bar{R}}\{0.25\}=\frac{2}{6}=\frac{1}{3}=0.33 \\
\gamma_{\bar{R}}\{0.33\}=\frac{6}{6}=1.00 & \gamma_{\bar{R}}\{0.50\}=\frac{3}{6}=\frac{1}{2}=0.50 \\
\gamma_{\bar{R}}\{0.67\}=\frac{2}{6}=\frac{1}{3}=0.33 & \gamma_{\bar{R}}\{0.83\}=\frac{0}{6}=0.00 \\
\gamma_{\bar{R}}\{1.00\}=\frac{0}{6}=0.00 &
\end{array}
$$

The plot given in Fig. 1, represents a discrete visualisation for when the descriptor is set to Red, all based on the returned degrees of significance. The membership value 0.33 returned 


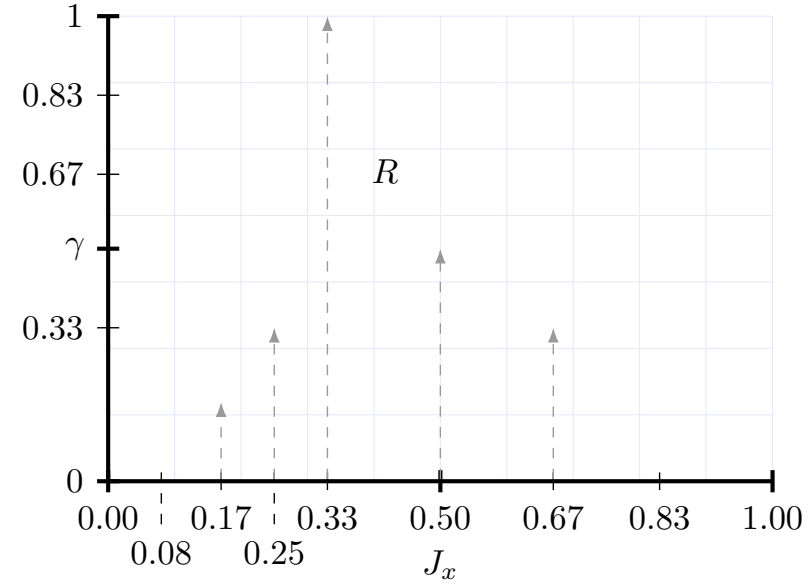

Fig. 1. A discrete visualisation for Red, based on the returned degrees of significance

a degree of significance of $\gamma \bar{R}\{0.33\}=1$, indicating that it was agreed upon unanimously. Fig. 1 represents this by having the apex of the stick height for 0.33 at the maximum value for significance, which is 1 . For any value to score a significance of 1 , it must satisfy the requirement stipulated by Eq. (10). The other membership values contained in the R-fuzzy set, in particular the upper approximation, all have their stick heights defined relative to their returned degrees of significance. The membership values that returned a degree of significance of 0 , these memberships were ignored as being valid candidates to represent the descriptor when set to Red. The closer the significance of a membership value is to 1 , the stronger its sentiment is agreed with. The closer to 0 , the less likely that it is to be treated as a valid candidate.

If one was inclined to create a set that encapsulated the degrees of significance for a specific descriptor, then one has inadvertently created the equivalence of a type-1 fuzzy set. Such are the similarities, the returned degree of significance will be equivalent to the degree of membership to the descriptor, if and only if the values for the significance degree provide the values for the membership function parameters. Fig. 2 provides a continuous interpretation for when the descriptor is set to Red. One can easily see that the degrees of significance would be the equivalent degrees of membership for all triggered membership values, as would be the case from a fuzzy perspective. The parameter values given were based on the apex height of the membership values' degree of significance, hence why they intersect through each apex. However, notice that a triangular membership function was not utilised, but instead a trapezoidal membership function was. This was done, as it seems more fitting that the values either side of a membership that scored a 1 for its significance would also score a 1 . As this is the continuous representation, and we know that $\gamma_{\bar{R}}\{0.33\}=1$, it is likely that 0.32 and 0.34 would also score a significance of 1 . By making use of a plateau for the function's apex, allows for a more realistic encapsulation. At what point does plateau taper off will be an arbitrary assumption, the chosen values for this instance were to have the plateau cover the interval $[0.3,0.45]$, hence why this is one possible continuous representation of the descriptor Red. By making use of the returned degrees of significance for

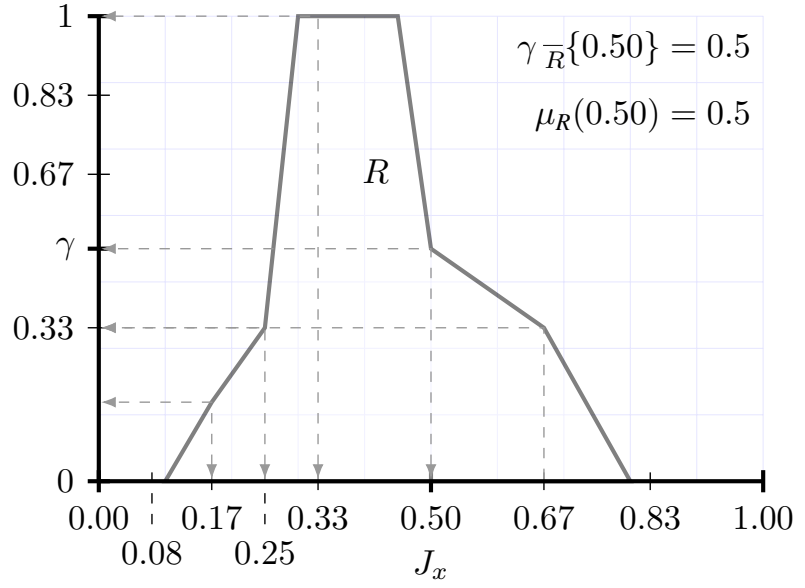

Fig. 2. A possible continuous visualisation for Red, based on the returned degrees of significance

a specific descriptor, one is able to create a set that encapsulates all associated membership values. This correlates correctly with each returned degree of significance and the equivalent fuzzy degree of membership, only if the parameters used to create the set, are based on the apex of the stick heights of the triggered membership values.

If one was to use a traditional type-1 fuzzy approach to define the membership Red, and attribute it to a single membership value, the average may be taken, in which case the result would be:

$$
\mu\left(f_{10}\right)=\frac{1}{14} \sum_{x \in \mathbb{U}} \mu(x) / x=0.39
$$

This value is based on the summation of each and every membership value contained in the generated subsets $M_{p j}\left(f_{10}\right) \in J_{x}$. The value itself 0.39 , is greater than the returned R-fuzzy value of 0.33 , meaning that the fuzzy value would be too skewed. If one was to apply an interval-valued fuzzy set to define the descriptor, utilising the most pessimistic lower bound and the most optimistic upper bound, one would be presented with an interval $[0.17,0.67]$. With this being the case, it is not possible to distinguish which values were agreed upon by all. The same problem would be the case for Atanassov intuitionistic fuzzy sets. As stated in Section I, the current extensions to fuzzy and its associated hybridisations, find it difficult to identify individual elements from their interval regions or shadow areas.

The plot given in Fig. 3, collectively presents a visualisation of all 3 R-fuzzy set descriptors. As one can see, the transition from one descriptor to the next conserves the degree of significance for each and every triggered membership value. For example the membership value 0.17 , scores a degree of significance of $\gamma \overline{D R}\{0.17\}=0.83$ when the descriptor is set to Dark Red. The same membership value scores a degree of significance of $\gamma \bar{R}\{0.17\}=0.17$, when the descriptor is set to Red. A single membership value can be interpreted differently depending on the descriptor that the R-fuzzy set is being modelled for. The plot also provides a means to understand the overall assumptions made by the populace. As it has been shown, the plots for each descriptor or perspective, 


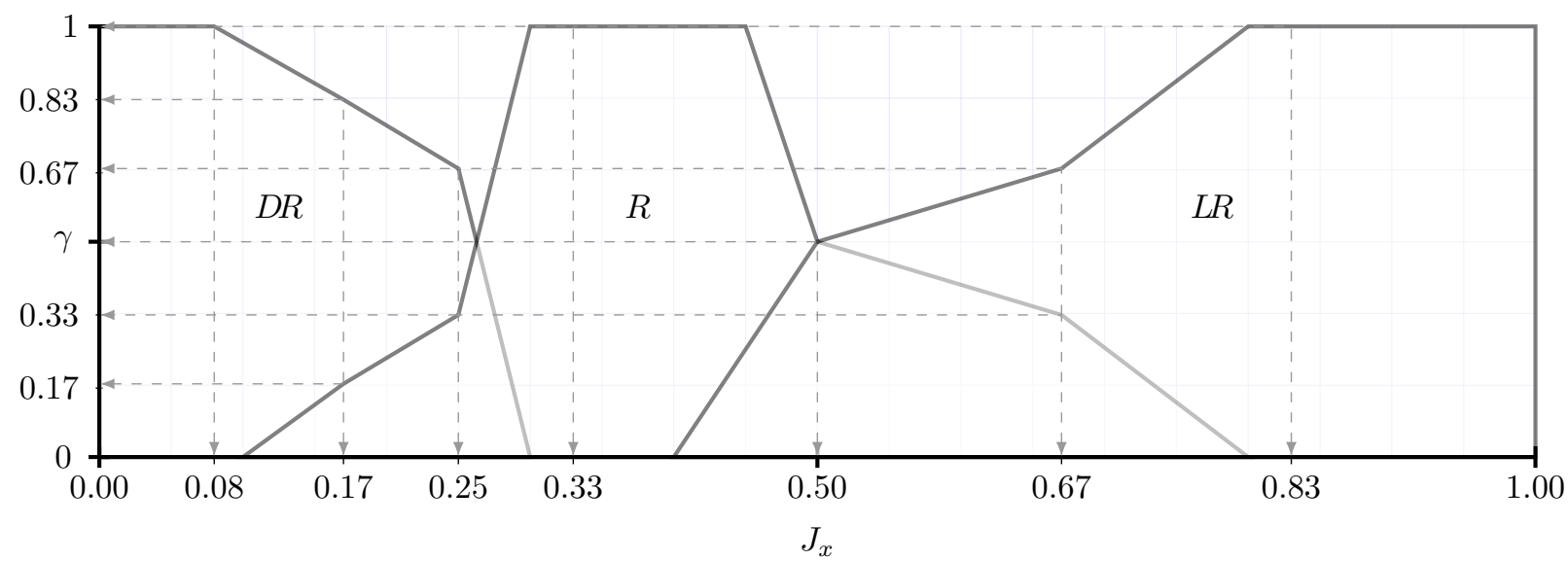

Fig. 3. A continuous visualisation for Example 1, based on all the generated significance measures for the R-fuzzy sets of Dark Red, Red \& Light Red

does not follow uniformed symmetry. This synthesis of human perception is more probable and in keeping, rather than the strictness associated with symmetrical uniformed sets.

\section{CONCLUSION}

This paper has presented a newly derived significance measure Eq. (9), one that quantifies the membership values contained within an R-fuzzy set. As it has been shown, an R-fuzzy approach for encapsulating uncertainty allows for a general consensus, and also specific held assumptions to be incorporated into a single R-fuzzy set. Current existing extensions to fuzzy and the various hybridised concepts, would still struggle to identify individual elements from their interval or shadow regions, the significance of the value itself would be lost. This is also true to some extent to the upper approximation of an R-fuzzy set, as for it to be included it merely has to be selected by at least one voter, or the vast majority but not all. This could effectively be a very large interval depending on the size of the populace; just creeping into the upper approximation would be not different to unanimously belonging there. The significance measure proposed uses the conditional probability of distribution relative to the generated subsets that the R-fuzzy is being modelled for. As Example 1 has demonstrated, understanding the importance of the membership values contained within the upper approximation allows for a better understanding on the perception being modelled. If a value just missed out on being included in the lower approximation, one may be inclined to treat it as such. Any such instance where human-based perception is being modelled, an R-fuzzy and significance degree coupling would provide for a powerful tool.

\section{REFERENCES}

[1] K. T. Atanassov, Intuitionistic fuzzy sets, Fuzzy Sets and Systems 20 (1) (1986) $87-96$.

[2] G. Cantor, Beitrge zur begrndung der transfiniten mengenlehre, Mathematische Annalen 46 (4) (1895) 481 - 512.

[3] D. Dubois, H. Prade, Rough fuzzy sets and fuzzy rough sets*, International Journal of General Systems 17 (2-3) (1990) 191-209.

[4] R. Jensen, Q. Shen, New approaches to fuzzy-rough feature selection, Fuzzy Systems, IEEE Transactions on 17 (4) (2009) 824-838.

[5] J. Mendel, R. John, Type-2 fuzzy sets made simple, Fuzzy Systems, IEEE Transactions on 10 (2) (2002) 117-127.
[6] S. Nanda, S. Majumdar, Fuzzy rough sets, Fuzzy Sets and Systems 45 (2) (1992) $157-160$.

[7] Z. Pawlak, Rough sets, International Journal of Computer \& Information Sciences 11 (5) (1982) 341-356.

[8] Z. Pawlak, Rough set theory and its applications to data analysis, Cybernetics \& Systems 29 (7) (1998) 661-688.

[9] Z. Pawlak, Rough sets and intelligent data analysis, Information Sciences 147 (14) (2002) 1-12.

[10] W. Pedrycz, Shadowed sets: representing and processing fuzzy sets, Systems, Man, and Cybernetics, Part B: Cybernetics, IEEE Transactions on 28 (1) (1998) 103-109.

[11] R. Sambuc, Fonctions $\Phi$ foues, Application a l'aide au Diagnostic en Patholologie Thyroidienne. PhD thesis, Univ. Marseille, France, 1975.

[12] Yingjie Yang, Chris Hinde, A new extension of fuzzy sets using rough sets: R-fuzzy sets, Information Sciences, Volume 180, Issue 3, 1 February 2010, Pages 354-365.

[13] L. A. Zadeh, Fuzzy sets, Information and control 8 (3) (1965) 338-353.

[14] L. Zadeh, The concept of a linguistic variable and its application to approximate reasoning-i, Information Sciences 8 (3) (1975) 199 - 249. 